\title{
A Regularized Boltzmann Scattering Operator for Highly Forward Peaked Scattering
}

\author{
Anil K. Prinja, PI \\ The University of New Mexico \\ Department of Chemical and Nuclear Engineering \\ Albuquerque, NM 87131, USA \\ prinja@unm.edu
}

\section{Introduction}

Extremely short collision mean free paths and near-singular elastic and inelastic differential cross sections (DCS) make analog Monte Carlo and deterministic computational approaches impractical for charged particle transport. The widely used alternative, the condensed history method, while efficient, also suffers from several limitations arising from the use of precomputed infinite medium distributions for sampling particle directions and energies. Accordingly, considerable attention has recently focused on the development of computationally efficient algorithms that implement the correct transport mechanics. Fokker-Planck [1] and Boltzmann Fokker-Planck [2] approximations have historically proved very useful in handling highly peaked scattering in certain classes of problems but these approaches are limited in the accuracy they can ultimately deliver. A more general methodology that allows accuracy to be systematically increased with practically no enhancement of algorithmic complexity has become possible with the advent of recently proposed higher order Fokker-Planck expansions [3] and their implementation in so-called Generalized Fokker-Planck models $[4,5,6]$. The goal of these newer approaches is to approximate the analog transport problem by one which is characterized by longer or stretched mean free paths and nonsingular collision operators but which can be solved numerically with considerably less effort than the analog problem and whose accuracy and efficiency can be readily adapted to a broad class of problems. One such implementation that has proved particularly efficient uses purely discrete scattering angle and hybrid discrete-continuous scattering angle representations [7, 8]. Moreover, generalizations of these methodologies to describe energy-loss straggling have been successfully demonstrated [6]. 
Here we describe an angular moment-preserving procedure to regularize the near-singular collision integral for electron elastic scattering by approximating and then re-summing what we refer to as a generalized Fermi expansion. It's difference from the generalized FokkerPlanck expansion of Leakeas and Larsen [4] will become apparent in the ensuing but we note here that the advantage of using the formulation developed below is that the resulting renormalized scattering kernel has a simple and explicit form which lends itself to a convenient implementation in Monte Carlo simulations. Furthermore, unlike our recent discrete scattering angle formulation [7,8], the present approach is continuous in scattering angle and consequently is free of ray artifacts. The generalized Fermi expansion is developed in the next section, followed by a presentation of the regularization procedure. Numerical results are then presented and the paper closes with some concluding remarks.

\section{Generalized Fermi Expansion}

Our approach consists of a modified use of earlier proposed higher-order Fokker-Planck expansions to describe scattering that is strongly anisotropic but not sufficiently forward peaked that a strictly Fokker-Planck approximation can be justified $[3,4,5]$. We note that this is invariably the situation for realistic electron scattering interactions such as described by the screened Rutherford cross section and its variants, but which nevertheless are nearsingular at zero deflection. As will become apparent shortly, our implementation amounts to approximating the angular diffusion Fokker-Planck operator, which is just the Laplacian on the unit sphere, by a planar Laplacian in this higher order expansion and it generalizes a result first due to Fermi [9].

We begin by writing the analog transport equation for the angular flux $\psi(\vec{r}, \vec{\Omega})$ of electrons at spatial position $\vec{r}$ along direction $\vec{\Omega}$ as

$$
\vec{\Omega} \cdot \nabla \psi(\vec{r}, \vec{\Omega})=J[\psi], \quad \psi\left(\vec{r}_{s}, \vec{\Omega}\right)=\delta\left(1-\vec{\Omega}_{0} \cdot \vec{\Omega}\right), \vec{n} \cdot \vec{\Omega}<0,
$$

where $\vec{n}$ is the outward directed unit normal at surface point $\vec{r}_{s}$ and $\vec{\Omega}_{0}$ is the incident beam direction. Also in Eq.(1), $J[\psi]$, a linear functional of the angular flux, is the elastic scattering collision integral, and is given by:

$$
J[\psi]=\int_{4 \pi} \sigma_{s}\left(\vec{\Omega}^{\prime} \cdot \vec{\Omega}\right) \psi\left(\vec{\Omega}^{\prime}\right) d \vec{\Omega}^{\prime}-\sigma_{s 0} \psi(\vec{\Omega})
$$

where $\sigma_{s}\left(\overrightarrow{\Omega^{\prime}} \cdot \vec{\Omega}\right)$ is the differential elastic scattering cross section (DCS), $\mu_{0}=\overrightarrow{\Omega^{\prime}} \cdot \vec{\Omega}$ is the cosine of the scattering angle, and $\sigma_{s 0}$ the corresponding inverse scattering mean free path. Energy losses are neglected in the present investigation but it has been demonstrated before that singular inelastic energy-loss operators can also be regularized [6].

Since charged particle interactions are mediated by long range Coulomb forces, the DCS $\sigma_{s}\left(\overrightarrow{\Omega^{\prime}} \cdot \vec{\Omega}\right)$ falls very sharply away from $\vec{\Omega}^{\prime} \cdot \vec{\Omega}=1$, decreasing in a continuous manner by several orders of magnitude over a scattering angle range of only a few degrees. This has the consequence that an initially peaked distribution will diffuse slowly in angle with increasing 
depth into the material and it enables us to introduce two simplifying approximations. First, we write

$$
\overrightarrow{\Omega^{\prime}} \cdot \vec{\Omega} \approx 1-\frac{1}{2}\left[\left(\eta-\eta^{\prime}\right)^{2}+\left(\xi-\xi^{\prime}\right)^{2}\right]
$$

where $\eta$ and $\xi$ are the $x$ and $y$ direction cosine components of $\vec{\Omega}$. Second, we extend the domain of $\eta$ and $\xi$ to the open interval $(-\infty, \infty)$, a reasonable step since the angular flux decays rapidly away from the initial direction. Using these results in Eq.(2) gives after some rearrangement

$$
J[\psi]=\int_{-\infty}^{\infty} \int_{-\infty}^{\infty} \hat{\sigma}_{s}\left(\eta^{2}+\xi^{2}\right) \psi\left(\eta-\eta^{\prime}, \xi-\xi^{\prime}\right) d \eta^{\prime} d \xi^{\prime}-\sigma_{s 0} \psi(\eta, \xi)
$$

where $\hat{\sigma}_{s}\left(\eta^{2}+\xi^{2}\right) \equiv \sigma_{s}\left[1-\left(\eta^{2}+\xi^{2}\right) / 2\right]$. Our goal is to represent Eq.(4) as a differential expansion and to that end we introduce the two dimensional Fourier transform pair

$$
\begin{gathered}
\bar{\psi}(k, l)=\frac{1}{2 \pi} \int_{-\infty}^{\infty} \int_{-\infty}^{\infty} e^{-i \vec{K} \cdot \vec{\alpha}} \psi(\eta, \xi) d \eta d \xi \\
\psi(\eta, \xi)=\frac{1}{2 \pi} \int_{-\infty}^{\infty} \int_{-\infty}^{\infty} e^{i \vec{K} \cdot \vec{\alpha}} \bar{\psi}(k, l) d k d l
\end{gathered}
$$

where $\vec{\alpha}(\eta, \xi)$ and $\vec{K}(k, l)$ are angle and transform vectors, respectively, and $\vec{K} \cdot \vec{\alpha}=(k \eta+l \xi)$. Noting the convolution structure of Eq.(4), the latter readily transforms to

$$
\bar{J}=\left[\bar{\sigma}\left(K^{2}\right)-\sigma_{s 0}\right] \bar{\psi}(k, l),
$$

where $K^{2}=\vec{K} \cdot \vec{K}$. Here and in the ensuing, appropriate behaviour of $\psi$ and its derivatives is assumed in order to justify the use of integral transforms. We further find it useful to express the Fourier transform of the differential cross section $\bar{\sigma}\left(K^{2}\right)$ as a Hankel transform, namely

$$
\bar{\sigma}\left(K^{2}\right)=\int_{0}^{\infty} d \lambda \lambda J_{0}(K \lambda) \hat{\sigma}_{s}\left(\lambda^{2}\right)
$$

where $J_{0}(\cdot)$ is the zeroth order Bessel function of the first kind. Proceeding, the infinite series representation of $J_{0}$ is used in Eq.(8) to express $\bar{\sigma}$ as an expansion in $K^{2}$ and the result inserted into Eq.(7). We next take a formal inverse Fourier transform of this equation to get, after some considerable algebra, the desired differential representation of the collision integral

$$
J[\psi]=\sum_{n=1}^{\infty} a_{n} \sigma_{n} \mathcal{L}^{n} \psi(\eta, \xi)=a_{1} \sigma_{1} \mathcal{L} \psi+a_{2} \sigma_{2} \mathcal{L}^{2} \psi+O\left(a_{3} \sigma_{3}\right) .
$$

In the above expression, the $a_{n}$ are positive numerical coefficients, $\mathcal{L}$ is the $2 \mathrm{D}$ planar Laplacian defined by

$$
\mathcal{L} \equiv \frac{\partial^{2}}{\partial \eta^{2}}+\frac{\partial^{2}}{\partial \xi^{2}}
$$


and we have introduced momentum transfer moments defined by

$$
\sigma_{n}=2 \pi \int_{-1}^{1}\left(1-\mu_{0}\right)^{n} \sigma_{s}\left(\mu_{0}\right) d \mu_{0}=2^{-n} 2 \pi \int_{0}^{\infty} d \lambda \lambda^{2 n+1} \hat{\sigma}_{s}\left(\lambda^{2}\right)
$$

We note in passing that Eq.(9), with $\mathcal{L}$ replaced by the Laplacian on the unit sphere, is related, but not identical, to the higher order Fokker-Planck expansion introduced by Pomraning [3] and subsequently used by Leakeas and Larsen [4] to develop a generalized FokkerPlanck approximation for the scattering integral. If scattering is sufficiently forward peaked, the $\sigma_{n}$ will form a rapidly decreasing sequence and truncation of the expansion in $\operatorname{Eq}(9)$ is suggested. It is especially interesting to consider the approximation to the collision integral that results from retaining only the first term in this sequence, namely

$$
J[\psi] \approx \frac{\sigma_{t r}}{2}\left(\frac{\partial^{2} \psi}{\partial \eta^{2}}+\frac{\partial^{2} \psi}{\partial \xi^{2}}\right)
$$

where $\sigma_{t r} \equiv \sigma_{1}$ is the familiar transport cross section. If, additionally, a thin target is assumed, throughout which the angular distribution remains nearly collimated, the resulting transport equation may be simplified to read

$$
\frac{\partial \psi}{\partial z}+\eta \frac{\partial \psi}{\partial x}+\xi \frac{\partial \psi}{\partial y}=\frac{\sigma_{t r}}{2}\left(\frac{\partial^{2} \psi}{\partial \eta^{2}}+\frac{\partial^{2} \psi}{\partial \xi^{2}}\right)
$$

with boundary condition

$$
\psi(x, y, 0, \eta, \xi)=\delta(x) \delta(y) \delta(\eta) \delta(\xi)
$$

This is the celebrated Fermi model, originally derived on physical grounds in the context of cosmic ray penetration of the atmosphere [9] and which has the following closed form joint Gaussian solution

$$
\psi(x, y, z, \eta, \xi)=\frac{3}{\pi^{2} \sigma_{t r}^{2} z^{4}} \exp \left[-\frac{2}{\sigma_{t r}}\left\{\frac{3\left(x^{2}+y^{2}\right)}{z^{3}}-\frac{3(x \eta+y \xi)}{z^{2}}+\frac{\left(\eta^{2}+\xi^{2}\right)}{z}\right\}\right] .
$$

An energy dependent generalization of this result in the continuous slowing down approximation forms the basis of Fermi-Eyges theory that is widely used but particularly so in electron dose calculations in radiotherapy [11]. The simplicity of this solution is undoubtedly a very attractive feature, but its accuracy is strictly limited to a small region around the pencil beam axis. The error increases dramatically with increasing lateral distance away from the axis and this deficiency in the solution can be traced to the neglect of large angle scattering which is manifested through the neglect of all momentum transfer moments higher than the first. While this conclusion suggests that greater accuracy may be achieved with the retention of additional terms in the expansion, more detailed theoretical analysis shows that in fact all truncations beyond the first term lead to unstable or unbounded solutions, in then sense of transforms at least. A similar conclusion was reached with the use of higher 
order Fokker-Planck expansions [4, 5]. The infeasibility of adopting higher order truncations eliminates the possibility of systematically obtaining closed form generalizations of the Fermi solution (however, see [10]). It is necessary to then consider approximations which require numerical solution methods but prove more efficient to solve than the analog problem. The considerations of this section are still very relevant, however, for the equivalence of the Boltzmann and differential forms of the collision operator, namely Eqs.(2) and (9), strongly suggests that approximate models which correctly reproduce a certain number of the cross section angular moments defined in Eq.(11) are likely to be more accurate than those that do not. This principle underlies the moment-preserving approach described in the next section.

\section{Regularized Collision Operator}

Consider the following functional of the angular flux

$$
G[\psi]=A \exp (\beta \mathcal{L}) \psi-A \psi
$$

where $\mathcal{L}$ is the $2 \mathrm{D}$ planar Laplacian defined above and $A$ and $\beta$ are unspecified constants. Expanding the exponential up to third order yields

$$
G[\psi]=A \beta \mathcal{L} \psi+\frac{A \beta^{2}}{2} \mathcal{L}^{2} \psi+O\left(A \beta^{3}\right)
$$

Comparing Eqs.(9) and (17), it follows that the $J$ - and $G$-functionals can be made formally equivalent through second order if $A$ and $\beta$ are chosen to satisfy

$$
A=2 \frac{\sigma_{1}^{2}}{\sigma_{2}}, \quad \beta=\frac{1}{4} \frac{\sigma_{2}}{\sigma_{1}},
$$

so that we can write

$$
J=G+O\left(\sigma_{2}^{2} / 32 \sigma_{1}+\sigma_{3} / 288\right)
$$

Thus, the two formulations of the scattering integral give identical first and second momentum transfer moments. The higher order terms will differ, of course, but if scattering is sufficiently forward peaked $\left(\sigma_{1} \gg \sigma_{2} \gg \sigma_{3} \cdots\right)$ the $G$-functional should represent an accurate approximation to the analog scattering integral. In particular, under circumstances where the Fokker-Planck approximation is accurate, the $G$-functional should also be accurate. Moreover, because the latter retains all higher order moments, although approximately, it has the potential of being more accurate than the Fokker-Planck approximation. We now demonstrate the significance of the $G$-functional formulation. Consider the quantity

$$
\chi(s, \eta, \xi)=\exp (s \mathcal{L}) \psi(\eta, \xi)
$$

where $s$ is a continuous non-negative real variable. With this definition, Eq.(16) can alternatively be written as

$$
G[\psi]=A \chi(\beta, \eta, \xi)-A \psi
$$


Now, differentiating Eq.(20) with respect to $s$ it is not difficult to show that $\chi$ satisfies the following "time dependent, infinite medium heat conduction" equation

$$
\frac{\partial \chi}{\partial s}=\frac{\partial^{2} \chi}{\partial \eta^{2}}+\frac{\partial^{2} \chi}{\partial \xi^{2}}, \quad s \geq 0, \quad-\infty<\eta, \xi<\infty
$$

with auxiliary data

$$
\chi(0, \eta, \xi)=\psi(\eta, \xi), \quad \lim _{|\eta|,|\xi| \rightarrow \infty} \chi(s, \eta, \xi)=0 .
$$

This initial-boundary value problem can be readily solved to obtain

$$
\chi(s, \eta, \xi)=\frac{1}{4 \pi s} \int_{-\infty}^{\infty} \int_{-\infty}^{\infty} \exp -\frac{\left[\left(\eta-\eta^{\prime}\right)^{2}+\left(\xi-\xi^{\prime}\right)^{2}\right]}{4 s} \psi\left(\eta^{\prime}, \xi^{\prime}\right) d \eta^{\prime} d \xi^{\prime}
$$

Finally, setting $s=\beta$ in Eq.(24) and inserting the result into Eq.(21) yields the following alternate representation of the $G$-functional

$$
\begin{aligned}
J[\psi] \approx G[\psi]= & \frac{A}{4 \pi \beta} \int_{-\infty}^{\infty} \int_{-\infty}^{\infty} \exp -\frac{\left[\left(\eta-\eta^{\prime}\right)^{2}+\left(\xi-\xi^{\prime}\right)^{2}\right]}{4 \beta} \psi\left(\eta^{\prime}, \xi^{\prime}\right) d \eta^{\prime} d \xi^{\prime} \\
& -A \psi(\eta, \xi)
\end{aligned}
$$

For obvious reasons, we refer to the above representation of the scattering integral as a moment-preserving approximation. It can form the basis for generalizing Fermi-Eyges theory to account for the effects of large angle scattering on electron dose deposition, and we hope to report on this in a future communication. Before we comment further on this result, however, we first note that Eq.(25) may be expressed in a form that is more practical for numerical implementation by restoring the domain of the direction variable to the unit sphere. This immediately follows upon using Eq.(3), getting

$$
J[\psi]=\frac{A}{4 \pi \beta} \int_{4 \pi} \exp \left[-\frac{\left(1-\mu_{0}\right)}{2 \beta}\right] \psi\left(\vec{\Omega}^{\prime}\right) d \vec{\Omega}^{\prime}-A \psi(\vec{\Omega}) .
$$

It should be noted that in so restricting the domain an error of $O(\exp (-1 / \beta))$ is introduced in the cross section moments. Although the coefficients can be rescaled to restore the exact moments, this is not necessary since $\beta$ is typically a small number. That is, the error is exponentially small and has no practical consequence.

There are a number of attractive features about the approximate collision integral obtained above and its generalization described below. First, we observe that the scattering operator has been regularized, i.e., the singularity at forward directions has been eliminated, and there is a concomitant reduction in the magnitude of the total scattering cross section. This smoothed approximation of the transport process greatly improves prospects for application of standard deterministic and Monte Carlo solution techniques. Also, the direct use of existing neutral particle transport codes becomes a distinct possibility since the algorithmic modifications necessary when differential Fokker-Planck approximations are employed are 
not required with the present formulation. Second, the approach presented here yields a univeral differential cross section that depends only on cross section angular moments. That is, the final result is independent of the detailed form of the underlying analog cross section, making it feasible to handle empirical or semi-empirical cross section represenations with relative ease. Finally, we note that sampling deflection angles from the exponential kernel in a Monte Carlo implementation is straightforward. This is in contrast to the infinite spherical harmonics expansion representation that results from the use of a generalized Fokker-Planck expansion [4].

Our approach can be readily extended to preserve higher moments of the analog DCS by generalizing Eq.(16) as follows

$$
G[\psi]=\sum_{n=1}^{N} A_{n} \exp \left(\beta_{n} \mathcal{L}\right) \psi-\left(\sum_{n=1}^{N} A_{n}\right) \psi
$$

The $2 N$ free parameters $\left\{A_{n}, \beta_{n}, n=1,2 \ldots N\right\}$ can be selected by requiring the consolidated generalized Fermi expansion of Eq.(27) to be identical to that of the analog expansion up to the appropriate order, thereby ensuring that the first $2 \mathrm{~N}$ momentum transfer moments of the DCS are preserved. This procedure will yield a nonlinear system of algebraic equations for the expansion parameters which in general must be solved numerically. Retaining higher order terms enables larger scattering angle effects to be accommodated although experience with other moment preserving strategies [7, 8] indicates that in practice two terms (four moments) is usually sufficient to accurately capture angular distributions even for relatively thin targets. However, a more effective way of incorporating large scattering angle effects is to first extract from the analog differential cross section a component that is not forward peaked and is characterized by a long mean free path. By further requiring the shape and amplitude of this smooth cross section to be identical to or closely resemble the analog cross section, high order moments can be accurately reproduced and large angle scattering effectively captured. The residual component will carry the singular part of the cross section and is more suitable for the regularization procedure described above. We have previously implemented this hybrid procedure with a discrete scattering angle representation of the residual cross section and observed the approach to be very accurate indeed [8].

\section{Numerical Results}

Monte Carlo simulations have been performed for $1 \mathrm{MeV}$ electrons incident on a gold target of thickness $T=3.85 \times 10^{-3} \mathrm{~cm}$ [8], and we compare transmitted and reflected angular distributions obtained using our new regularized model, in pure and hybridized form, and our previous discrete scattering angle representation [7] against corresponding benchmark analog results. The screened Rutherford scattering cross section [7] was used for sampling angular deflections in the analog model and for computing the necessary momentum transfer moments. Energy dependence was neglected in these simulations so that the electrons were either transmitted or reflected from the infinite slab. 
In Figure 1, the relative differences with analog results are shown for the 1- and 2discrete angle model and the exponential kernel model. The absence of ray artifacts with our regularized model stands in stark contrast to the discrete models. The exponential model is less accurate than the 2-discrete angle model near grazing angles for reflection, but this can partly be attributed to the discrete model preserving two additional moments. Because of the greater expense of sampling from an exponential distribution, our new model is only slightly faster than the 2-discrete angle method. The discrete model shows ray effects
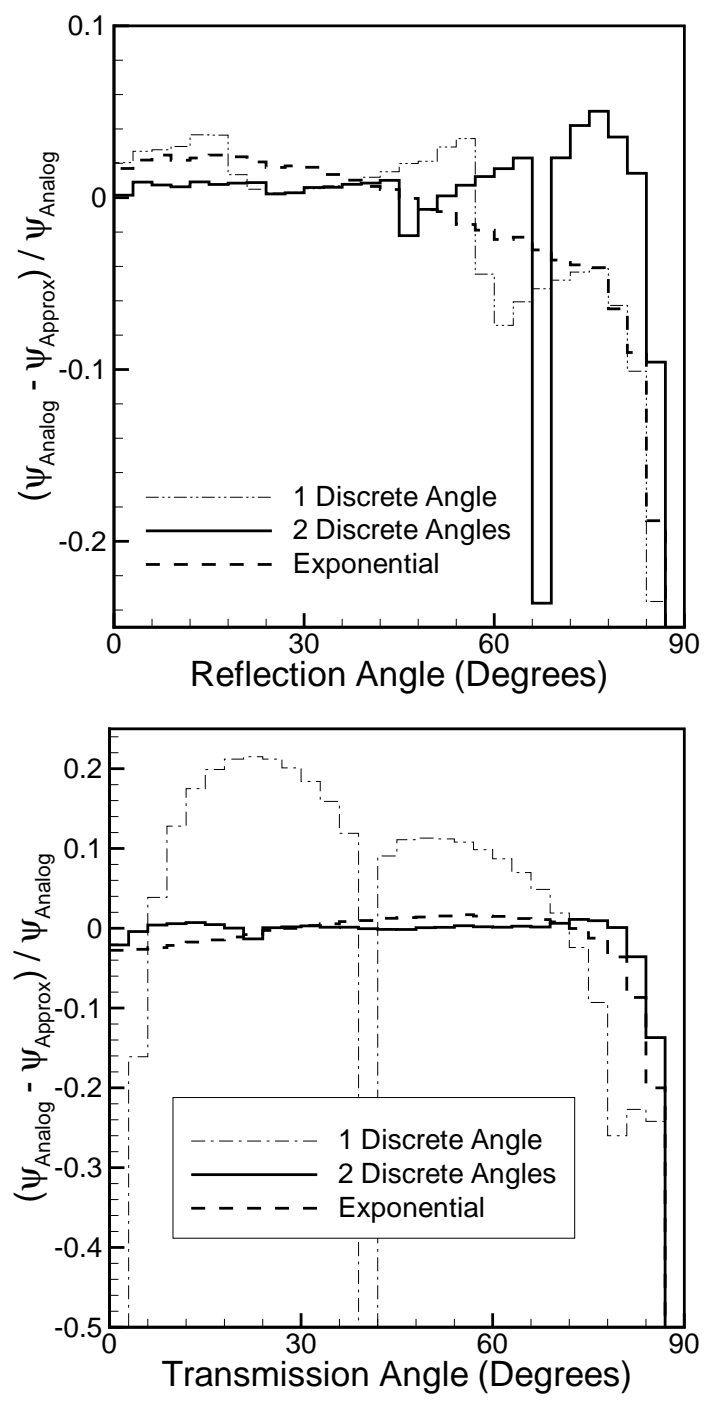

Figure 1: Error in Angular Escape Distributions Relative to Outward Normal for Electrons Incident on a Gold Slab for Discrete and Exponential Kernels.

in the reflected angular distribution due to the presence of a discrete scattering angle in the backward direction while the exponential model yields a smooth distribution across all 
angles, as expected. Both methods perform well in calculating the transmitted distribution, with the discrete method performing better at large angles relative to the surface normal. The 2 discrete angle method accurately captures four angular scattering moments, while the exponential model accurately captures only two moments. Because of the relative size of the interaction cross section and the greater expense of sampling from an exponential distribution, the exponential model is only slightly faster than the 2 angle method.

In Figure 2, the relative differences with analog results are shown for the exponential model and the hybrid model. The hybrid model shows greatly improved accuracy for both transmitted and reflected distributions, a consequence of more accurately treating the higher order momentum transfer moments. However, this improved accuracy is achieved at a slightly increased computational expense, with the runtime for the hybrid model approximately two times greater than for the pure exponential model.

\section{Conclusions}

We have demonstrated that a closed form regularized scattering kernel for electron transport, derived using an angular moment-preserving technique, yields accurate escape distributions. Unlike our previous discrete scattering angle representation, the present model is free of ray artifacts yet it is computationally competitive. Moreover, the procedure described herein can be readily and systematically generalized to incorporate more accurate physics, withough increasing algorithmic complexity, by retaining successively higher order angular moments of the analog differential cross section. Finally, numerical testing shows that the best performance, from accuracy and computational efficiency considerations, is realized when the cross section is first decomposed into smooth, analog-like and singular components and the regularization procedure applied to the latter.

\section{References}

[1] J. E. Morel, "Fokker-Planck Calculations Using Standard Discrete Ordinates Codes," Nucl. Sci. Eng., 79, 340 (1981).

[2] M. Caro and J. Ligou, " Treatment of Scattering Anisotropy of Neutrons Through the Boltzmann Fokker-Planck Equation," Nucl. Sci. Eng., 83, 242 (1983).

[3] G. C. Pomraning, "Higher Order Fokker-Planck Operators," Nucl. Sci. Eng., 124, 390 (1996).

[4] C. L. Leakeas and E. W. Larsen, "Generalized Fokker-Planck Approximations of Particle Transport with Highly Forward-Peaked Scattering," Nucl. Sci. Eng., 137, 236 (2001).

[5] A. K. Prinja and G. C. Pomraning, "A Generalized Fokker-Planck Model for Transport of Collimated Beams," Nucl. Sci. Eng., 137, 227 (2001). 

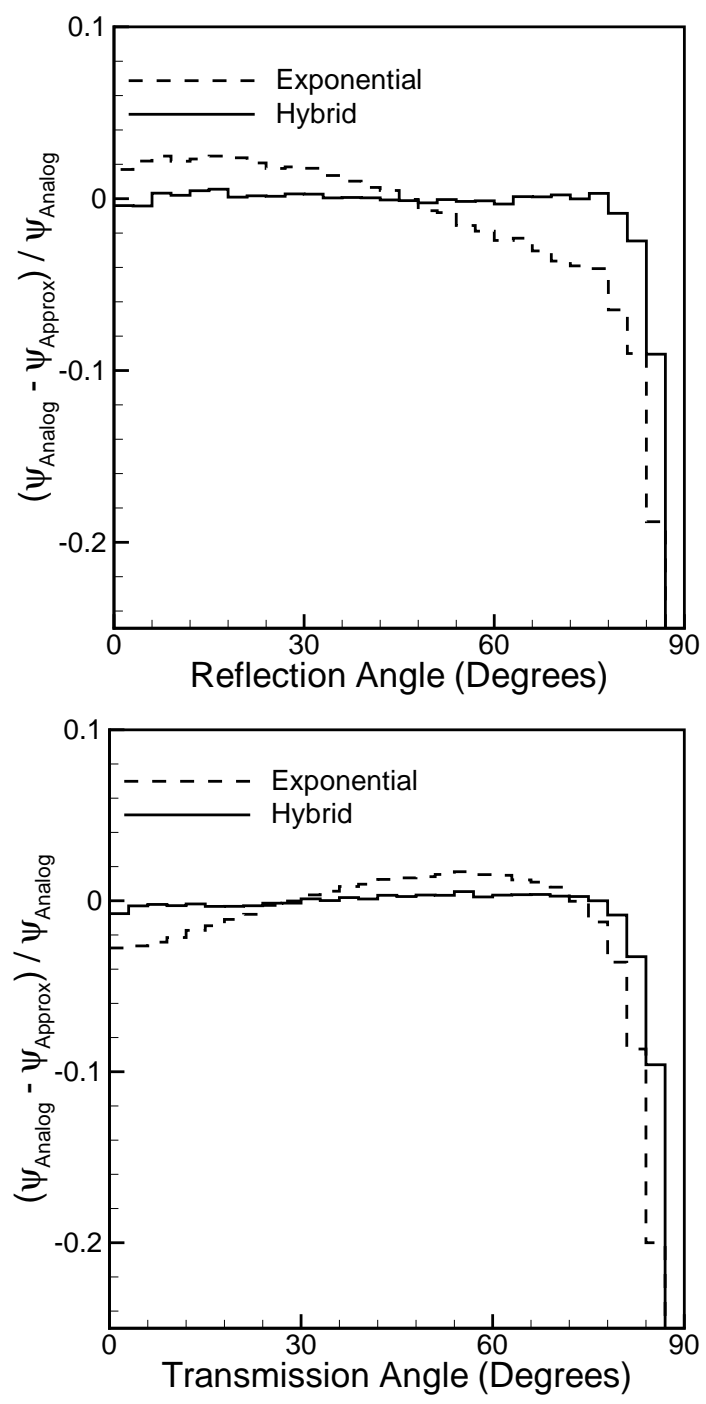

Figure 2: Error in Angular Escape Distributions Relative to Outward Normal for Electrons Incident on a Gold Slab for Exponential Kernels.

[6] A. K. Prinja, V. M. Klein and H. G. Hughes, "Moment Based Effective Transport Equations for Energy Straggling," Trans. Am. Nucl. Soc., 86, 204 (2002).

[7] B. C. Franke, A. K. Prinja, R. P. Kensek and L. J. Lorence, "Discrete Scattering-Angle Model for Electron Pencil Beam Transport," Trans. Am. Nucl. Soc., 86, 206 (2002).

[8] B. C. Franke, A. K. Prinja, R. P. Kensek and L. J. Lorence, "Ray Effect Mitigation for Electron Transport with Discrete Scattering-Angles," Trans. Am. Nucl. Soc., 87, 133 (2002). 
[9] B. Rossi and K. Greisen, "Cosmic Ray Theory," Rev. Modern Phys., 13, 240 (1941).

[10] G. C. Pomraning and A. K. Prinja, "The Pencil Beam Problem for Screened Rutherford Scattering," Nucl. Sci. Eng., 130,1 (1998).

[11] K. R. Hogstrom, M. D. Mills and P. R. Almond, "Electron Beam Dose Calculations," Phys. Med. Biol., 26, 445 (1981) 